\title{
Crecimiento del Pinus radiata sometido a diferentes tratamientos de raleo y poda en el sudeste de la provincia de Buenos Aires, Argentina
}

\author{
Growth of Pinus radiata undergoing different thinning and pruning treatments \\ in the Southeast of the province of Buenos Aires, Argentina
}

\author{
Paula Ferrere ${ }^{\text {a*, Ana María Lupi }{ }^{\text {b }} \text {, Teresa Boca }}{ }^{\text {c }}$ \\ *Autor de correspondencia: a AER INTA 9 de Julio EEA Pergamino, Nueve de Julio, Provincia de Buenos Aires, Argentina, \\ Mitre 857 Nueve de Julio, Buenos Aires, Argentina, tel./fax: +0054 2317 431840, ferrere.paula@inta.gob.ar \\ ${ }^{\mathrm{b}}$ Instituto de Suelos, INTA Castelar, Villa Udaondo Castelar, Buenos Aires, Argentina. \\ c Instituto de Clima y Agua, INTA Castelar, Villa Udaondo Castelar, Buenos Aires, Argentina.
}

\begin{abstract}
SUMMARY
Silvicultural treatments aim at increasing productivity in intervention stands. Bearing in mind the lack of prior information, the goals of this study are to assess the growth of Pinus radiata in the Southeast of the province of Buenos Aires, Argentina, and to promote the implementation of early intense pruning and thinning, assessing tree behavior at the individual and stand level. The trial was installed in a plantation of six years, with initial density of 1,111 plants ha $^{-1}$, on a Hapludol $(<50 \mathrm{~cm}$ effective depth) Treatments studied were: T1, control treatment, without intervention; T2, $50 \%$ thinning, without pruning; T3, $75 \%$ thinning, without pruning; T4, $50 \%$ thinning, with pruning; and T5, $75 \%$ thinning, with pruning. The trial was carried out in a six-year plantation, and measured yearly until the trees reached 13 years of age. Studied variables were: quadratic mean diameter, basal area and volume. Thinning had a significant and immediate impact on the production of wood at the stand level, whereas pruning did not led to significant growth losses, when applied in equivalent thinning intensities. Quadratic mean diameter increased differentially, with increases in the most intense thinning treatments. After 13 years, the most significant volume was found in $\mathrm{T} 1\left(189 \mathrm{~m}^{3} \mathrm{ha}^{-1}\right)$. When the growth of the 200 dominant trees was analyzed, treatments with $75 \%$ thinning and with and without pruning differed statistically from the control. The intensity of $50 \%$ thinning six years after plantation is the most recommended since it allows combining growth at the individual tree with a significant accumulation of volume remaining trees. Considering these soils do not compete with agricultural uses, these are interesting options to be considered as complementary productions.
\end{abstract}

Key words: radiata pine, quadratic mean diameter, basal area, volume, forestry management.

\section{RESUMEN}

Los tratamientos silvícolas orientados a la producción de madera de calidad tienen como finalidad concentrar la producción volumétrica en los mejores individuos. Atendiendo a la falta de información local previa, el objetivo del presente estudio fue evaluar el crecimiento de árboles individuales y de rodales. El ensayo se instaló en una plantación de seis años, con densidad inicial de 1.111 plantas ha ${ }^{-1}$, sobre un Hapludol (<50 cm de profundidad efectiva). Los tratamientos fueron T1: testigo sin intervención; T2: $50 \%$ de raleo (\% del número total de plantas por hectárea), sin poda; T3: $75 \%$ raleo, sin poda; T4: $50 \%$ raleo, con poda y T5: $75 \%$ raleo, con poda. Se medió anualmente hasta el año 13: diámetro medio cuadrático $(d m c)$, área basal y volumen. A los 13 años el máximo volumen se observó en T1 (189,7 m $\left.\mathrm{ha}^{-1}\right)$. El raleo afectó significativamente la producción de madera en volumen a nivel de rodal, mientras que la poda sólo lo hizo en el tratamiento del $50 \%$ de raleo a los 13 años. El $d m c$ incrementó en forma diferencial, aumentando en los tratamientos de raleo más intensos. El crecimiento en $d m c$ de los 200 árboles dominantes fue significativamente mayor en los tratamientos con $75 \%$ de raleo (con y sin poda) que en el testigo. La intensidad de raleo del $50 \%$ a los seis años del establecimiento de la plantación es el tratamiento recomendado, porque permite combinar crecimiento a nivel de árbol individual con una acumulación importante de volumen en los árboles remanentes.

Palabras clave: pino insigne, diámetro medio cuadrático, área basal, volumen, manejo silvícola.

\section{INTRODUCCIÓN}

Pinus radiata D. Don es una de las principales especies forestales cultivadas a nivel mundial, estimándose actualmente unas cuatro millones de hectáreas implantadas (Mead 2013). Es originaria de una estrecha franja costera de California, pero su cultivo se ha difundido ampliamente, encontrándose en países como Chile, Nueva Zelanda, Portugal y España. En Argentina, las forestaciones se localizan en las zonas serranas de la provincia de Córdoba, en las sierras de Tandil, Sierras de Ventana y en el cordón dunoso de la costa atlántica, en la provincia de Buenos Aires; 
y en menor medida en algunos valles cordilleranos de la Patagonia Norte (Golfari 1959). Como característica destacable, se trata de una madera versátil, con aplicaciones estructurales y decorativas, tales como marcos, paredes, vigas, chapas y tableros. En los últimos años ha sumado a sus beneficios la utilización de estas plantaciones como sumideros de carbono y también para la producción de biomasa (Merino et al. 2003).

En Argentina en general, la silvicultura de las plantaciones de pino ha evolucionado en forma diferencial según las regiones del país. En la provincia de Misiones y nordeste de Corrientes, desde principios de la década del 90 se han difundido e implementado sistemas de manejos silvícolas intensivos para la producción de madera de alto valor (Crechi et al. 2001, Costas et al. 2007, Fassola et al. 2004). Por el contrario, en las zonas de serranías y en el cordón dunoso de la provincia de Buenos Aires, las forestaciones de Pinus radiata se caracterizan por la ausencia parcial o total de intervenciones silvícolas. El estado actual del patrimonio forestal, concentrado básicamente en medianos y grandes productores agrícolas, se presenta con plantaciones densas, sin raleos ni podas y, en consecuencia, con bajo valor comercial.

Para alcanzar el mercado de madera libre de nudos, el manejo silvícola se debe orientar a la producción de materia prima de calidad, concentrando el crecimiento en unos pocos árboles de buen tamaño, forma y con la menor cantidad posible de defectos; estándares que no son alcanzados por las plantaciones de Pinus radiata de media a avanzada edad localizadas en la provincia de Buenos Aires.

El desafío es intervenir los rodales jóvenes y para ello es necesario ajustar la combinación de tratamientos silvícolas en una determinada intensidad, en el momento oportuno, según la calidad de sitio (Gerding 1991, Espinosa et al. 1994, Trincado y Burkart 2009, Groot y Schenider 2011). De esta manera se mejorará calidad de la madera, aumentando los beneficios económicos (Fassola et al 2002).

La densidad del rodal, que puede ser determinada con el raleo, es una medida del espacio que el árbol tiene para crecer (Bravo et al. 2011) y se asocia directamente a la producción total y de madera libre de nudos (García 1993). La magnitud de la respuesta a tratamientos de raleo está determinada por la intensidad del mismo, el momento de aplicación en el rodal y la calidad de sitio (Zhang et al. 1997). Según Schoelzke (2003), las ganancias en crecimiento por la aplicación de raleos en Pinus elliottii Engelm. fueron del $30 \%$ en el diámetro y en un $96 \%$ en el área basal. Espinosa et al. (1994) trabajando con plantaciones de $P i$ nus radiata en Chile, encontraron, después de seis años, que parcelas sometidas a intensidades de raleo del 74, 61 y $48 \%$, crecieron significativamente más en diámetro que el testigo (1.550 árboles ha' ${ }^{-1}$, o con un raleo leve del 23 $\%$. En cambio, cuando se tomaron los 200 árboles ha ${ }^{-1}$ de mayor tamaño de cada tratamiento, no se encontraron diferencias en diámetro entre intensidades de raleo, y un comportamiento similar se observó con la variable altura.
La tendencia fue inversa cuanto se analizó el crecimiento de la masa, presentándose las mayores acumulaciones de área basal y volumen en las parcelas de menor intensidad de raleo. También Rodríguez et al. (2003), trabajando con Pinus radiata, encontraron árboles de mayor tamaño en sistemas con baja densidad (como los silvopastoriles) en relación a los sistemas forestales puros (con densidades de 1.600 plantas ha $^{-1}$ ). En rodales de Pinus taeda L., Zhang et al. (1997) encontraron que intensidades de raleo del $30 \mathrm{y}$ $50 \%$, tuvieron un efecto positivo tanto en el crecimiento en altura como en diámetro; sin embargo, el efecto del raleo sobre la altura fue mayor en sitios de buena calidad. La mayor incidencia del raleo sobre el diámetro que la altura, podría sugerir un cambio en la forma de los árboles según la calidad del sitio en que se encuentre el rodal. En Misiones, Argentina, en una plantación de Pinus taeda, Costas et al. (2002) probaron el efecto de intensidades de raleo del 49 y del $65 \%$, aplicados a los tres años de edad, en comparación con un testigo con 1.480 árboles $\mathrm{ha}^{-1}$. A los cinco años, el raleo más intenso mostró un mayor volumen de árbol individual, mientras que el volumen del rodal fue mayor en el tratamiento testigo. El testigo presentó el menor diámetro promedio y no se reportó una influencia significativa sobre la altura. Similares resultados se reportaron en Pinus halepensis Miller, por Navarro et al. (2010) y las diferencias persistieron a lo largo de 5 años.

La poda realizada en el momento adecuado permite obtener madera libre de nudos; sin embargo, su intensidad puede afectar la fisiología del árbol y, en consecuencia, el crecimiento (Pinkard y Beadle 2000). Las podas severas pueden influir negativamente sobre el crecimiento diamétrico perdurando el efecto hasta la edad de cosecha (Meneses y Guzmán 2000). Si se efectúa antes del cierre del dosel es posible que se produzcan pérdidas de crecimiento, debido a que las ramas podadas se encuentran activas. En cambio, si se realiza luego del cierre del dosel, estando las ramas sombreadas pero aun vivas; afectará poco al crecimiento del árbol (Barrio et al. 2009). Sutton y Crowe (1975) estudiaron el efecto de la remoción de la copa en porcentajes del 20, 35, 50 y $60 \%$. Para intensidades iguales o inferiores al $50 \%$ no se vio afectado el crecimiento en diámetro, en concordancia con Neilsen y Pinkard (2003) y Schoelzke (2003).

En Misiones, Argentina, Fassola et al. (2002) hallaron que variables como el diámetro, la altura y el volumen cilindrométrico de árboles de Pinus taeda fueron afectadas en distinto grado por la intensidad de los raleos y de las podas. Para intensidades de raleo del $70 \%$, porcentajes de remoción de copa del $30 \%$ no afectaron el crecimiento. Sin embargo, para intensidades de poda del 50 y $70 \%$ de remoción de copa, se afectó el crecimiento en diámetro y en menor medida la altura. En la misma región, Costas et al. (2005) probaron el efecto de cuatro niveles de alturas final de podas $(3,3 \mathrm{~m}, 4,4 \mathrm{~m}, 5,5 \mathrm{~m}$ y $6,6 \mathrm{~m})$. Los tratamientos con $3,30 \mathrm{~m}$ de altura final de poda presentaron los mayores diámetros promedios y volumen individual 
en comparación con los tratamientos que alcanzaron una altura de poda de $6,6 \mathrm{~m}$. Por otro lado, los tratamientos de poda no afectaron la altura media.

En la región pampeana y particularmente en el sector de sierras de Tandil, no se dispone de un esquema de manejo silvícola ajustado de tal manera que las plantaciones jóvenes o las nuevas forestaciones puedan generar productos de mayor valor comercial. Ante la carencia de antecedentes locales respecto de los regímenes de manejo más apropiados para Pinus radiata, se estableció un ensayo con el objetivo de analizar la incidencia de tratamientos silvícolas de poda y raleo sobre el crecimiento de diferentes variables dasométricas y estudiar su evolución por un período de siete años. La hipótesis de este trabajo es que la aplicación de podas del $40 \%$ de remoción de la copa a los seis años no afecta significativamente el crecimiento del árbol siendo el raleo el tratamiento silvícola que más influye sobre el crecimiento a nivel de árbol individual y de rodal.

\section{MÉTODOS}

Características del área de estudio. El ensayo se ubicó en una plantación forestal del Establecimiento San Gabriel, a unos $50 \mathrm{~km}$ de la localidad de Tandil, provincia de Buenos Aires, Argentina ( $37^{\circ} 33^{\prime} 34^{\prime \prime}$ S y $\left.59^{\circ} 13^{\prime} 01^{\prime \prime} \mathrm{O}\right)$. El experimento se localizó la ladera de un cerro, ocupando una posición de media loma a loma, con pendientes en torno al $5-10 \%$. El suelo del sitio del experimento es un Hapludol de color negro, de textura superficial franco limosa, muy somero, bien drenado, formado sobre sedimentos loéssicos y de aptitud ganadera (clase $\mathrm{VI}_{\mathrm{es}}$ ). El espesor del solum en el sitio del experimento puede variar desde los 10 en el sector más alto hasta los $50 \mathrm{~cm}$ en el piedemonte y se presentan afloramientos rocosos. El factor limitante es la profundidad efectiva del suelo que condiciona el anclaje de las raíces y la disponibilidad de agua. En general se trata de suelos sin uso previo, con altos contenidos de materia orgánica y buena fertilidad natural.

Clima. El clima de la zona se clasificó según Köppen-Geiger como $\mathrm{Cfb}$, definido como templado, con precipitaciones durante todos los meses del año y temperaturas medias del mes más cálido, inferior a los $22^{\circ} \mathrm{C}$. De acuerdo a lo reportado por Jackson y Gifford (1974), se encuentra dentro del óptimo para el cultivo del $P$. radiata, con temperaturas medias de $13,9^{\circ} \mathrm{C}$, mínima promedio de $7,7^{\circ} \mathrm{C}$ y máxima promedio de $20^{\circ} \mathrm{C}$ para la zona en estudio (INTA Castelar). La precipitación promedio para la serie analizada fue de $885 \mathrm{~mm}$, similar a la reportada para el clima mediterráneo de Romanyà y Vallejo (2004) y a la zona de Cauquenes en Chile (Álvarez et al. 2013). En la figura 1 se observan las oscilaciones de las precipitaciones (PP, mm) ocurridas desde el año 1980 hasta el año 2014.

Diseño de experimento y tratamientos. El ensayo se instaló en un sitio sin uso previo, en el otoño del año 2007 en una plantación realizada en el año 2001. La densidad inicial de plantación fue de 1.111 plantas ha-1 $^{-1}(3 \mathrm{~m} \times 3 \mathrm{~m})$ y recibió los tratamientos y cuidados culturales tradicionales de la región.

En el cuadro 1 se detallan los tratamientos estudiados que resultan de la combinación de dos intensidades de poda (sin poda y $40 \%$ de remoción de la copa viva) y

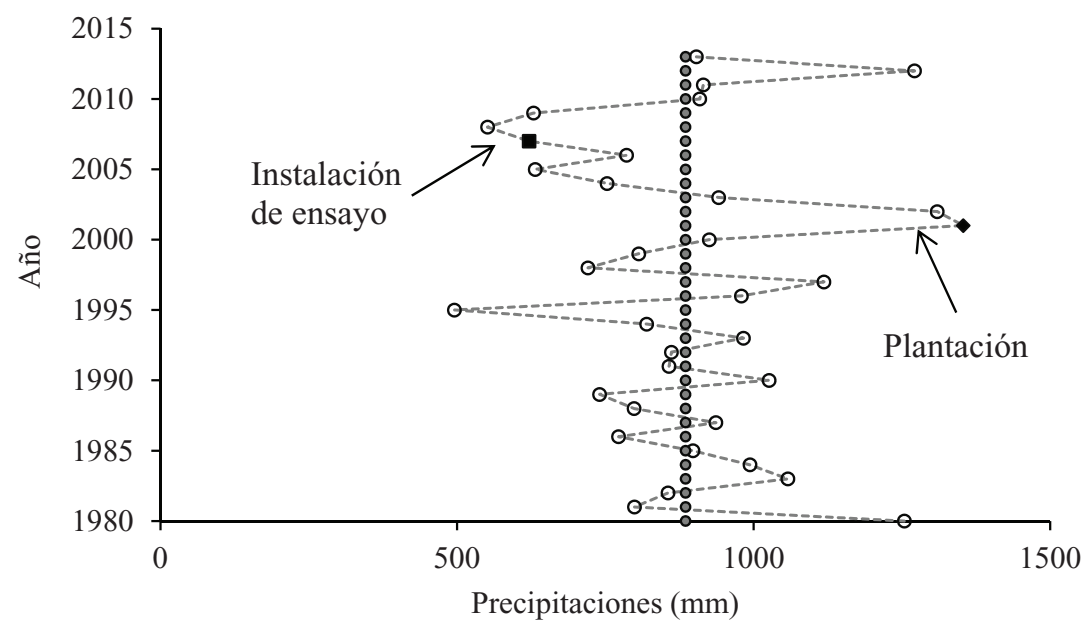

Figura 1. Variabilidad en las precipitaciones ocurridas en la zona del ensayo (Fuente: Instituto de Clima y Agua INTA Castelar). La línea vertical corresponde al promedio histórico. El punto negro del año 2000 (a la derecha de la línea vertical) corresponde al promedio anual de precipitaciones del año en que se realizó la plantación y el punto negro del año 2007 (a la izquierda de la línea vertical) corresponde al promedio del año en que se aplicaron los tratamientos.

Rainfall variation in the test area (source: INTA Castelar Climate and Weather Institute). The vertical line shows the historical average. The black spot in the year 2000 (to the right of the vertical line) shows the annual average rainfall of the year of the plantation, while the black spot in 2007 (to the left of the vertical line) shows the average rainfall for the year of the treatments. 
Cuadro 1. Tratamientos aplicados en el ensayo de Pinus radiata y estado dasométrico anterior y posterior a la instalación del ensayo. Applied treatments in the Pinus radiata trial and dasometric state, before and after trial installation.

\begin{tabular}{|c|c|c|c|c|c|c|c|}
\hline \multirow{3}{*}{ Tratamientos } & \multicolumn{3}{|c|}{ Estado del rodal pre-tratamientos } & \multicolumn{4}{|c|}{ Estado del rodal post-tratamientos } \\
\hline & $d m c$ & área basal & volumen & $\mathrm{N}$ & $d m c$ & área basal & volumen \\
\hline & $(\mathrm{cm})$ & $\left(\mathrm{m}^{2} \mathrm{ha}^{-1}\right)$ & $\left(\mathrm{m}^{3} \mathrm{ha}^{-1}\right)$ & (árboles ha-1) & $(\mathrm{cm})$ & $\left(\mathrm{m}^{2} \mathrm{ha}^{-1}\right)$ & $\left(\mathrm{m}^{3} \mathrm{ha}^{-1}\right)$ \\
\hline \multirow{2}{*}{$\mathrm{T} 1: \sin$ poda, sin raleo } & 12,6 & 12,8 & 35,8 & \multirow{2}{*}{1.033} & 12,6 & 12,8 & 35,8 \\
\hline & $( \pm 0,3)$ & $( \pm 1,2)$ & $( \pm 7,6)$ & & $( \pm 0,3)$ & $( \pm 1,2)$ & $( \pm 7,6)$ \\
\hline \multirow{2}{*}{$\mathrm{T} 2: \sin$ poda, $50 \%$ raleo } & 11,5 & 12 & 44,3 & \multirow{2}{*}{546} & 13,1 & 7,4 & 28,2 \\
\hline & $( \pm 1,8)$ & $( \pm 3,8)$ & $( \pm 15,9)$ & & $( \pm 0,7)$ & $( \pm 1,1)$ & $( \pm 2,8)$ \\
\hline \multirow{2}{*}{ T3: sin poda, $75 \%$ raleo } & 10,9 & 10,7 & 38,7 & \multirow{2}{*}{333} & 12,5 & 3,5 & 13,2 \\
\hline & $( \pm 1,0)$ & $( \pm 2,5)$ & $( \pm 10,1)$ & & $( \pm 1,5)$ & $( \pm 1,0)$ & $( \pm 4,5)$ \\
\hline T4: $40 \%$ poda, $50 \%$ raleo & 11,8 & 11,8 & 43,6 & \multirow{2}{*}{533} & 12,9 & 7,1 & 27,1 \\
\hline Altura de poda: $5,16 \mathrm{~m}$ & $( \pm 0,4)$ & $( \pm 1,7)$ & $( \pm 6,7)$ & & $( \pm 0,4)$ & $( \pm 0,6)$ & $( \pm 2,6)$ \\
\hline T5: $40 \%$ poda, $75 \%$ raleo & 11,4 & 10,7 & 43,2 & \multirow{2}{*}{283} & 13,1 & 3,9 & 14,8 \\
\hline Altura de poda: $4,83 \mathrm{~m}$ & $( \pm 1,4)$ & $( \pm 3,1)$ & $( \pm 10,3)$ & & $( \pm 0,9)$ & $( \pm 0,5)$ & $( \pm 2,2)$ \\
\hline
\end{tabular}

Valores medios \pm desvío estándar. $d m c$ : diámetro métrico cuadrático.

tres intensidades de raleo (sin raleo, $50 \%$ y $75 \%$, como porcentaje de árboles cortados del inicialmente plantado). Debido a la inclinación del terreno se aplicó un diseño en bloques completos al azar con cuatro repeticiones.

El raleo se aplicó por única vez en abril de 2007. Los tratamientos de poda se aplicaron secuencialmente en el otoño del año 2007, 2009 y 2010 y la remoción del $40 \%$ de la copa significó, en promedio, una altura de inicio de copa de 4,98 m ( $\pm 0,74 \mathrm{~m})$. La superficie de cada parcela fue de $600 \mathrm{~m}^{2}$ y cada una tenía una fila de bordura. La superficie total del ensayo fue de 1,8 ha.

Mediciones y análisis de los datos. Previo a la aplicación de los tratamientos de intensidad de raleo se realizó un relevamiento del diámetro a la altura del pecho (dap) de todos los individuos de las parcelas y su estado sanitario y sociológico. En el cuadro 1 se muestran el estado de las variables dasométricas previo a la aplicación de los tratamientos. A partir de esta medición se definieron los individuos a eliminar según la intensidad de raleo correspondiente. Se priorizó la eliminación de árboles dominados, de mala forma y enfermos. Se realizaron mediciones anuales del dap en todos los árboles de las parcelas, desde el año 6 (luego de aplicar los tratamientos) al año 13.

Para la estimación de los volúmenes individuales se asumió un modelo logarítmico desarrollado para la región ${ }^{1}$, cuya forma es la siguiente: $\ln \mathrm{v}_{\mathrm{i}}=-9,13181+2,39432 * \ln$ (dap). Se calculó el diámetro cuadrático promedio $(d m c$, $\mathrm{cm})$, el área basal $\left(G, \mathrm{~m}^{2} \mathrm{ha}^{-1}\right)$ y el volumen $\left(\mathrm{V}, \mathrm{m}^{3} \mathrm{ha}^{-1}\right)$, siendo estos estimadores de la productividad del rodal. Es-

\footnotetext{
Ferrere et al. 2009, no publicado.
}

tas mismas variables fueron calculadas para los 200 árboles de mayor diámetro por hectárea. Se analizaron los incrementos corrientes anuales entre períodos de medición para las siguientes variables: diámetro medio cuadrático $(d m c)$ $\left(\operatorname{ICA}_{d m c}, \mathrm{~cm} \mathrm{ha} a^{-1} \mathrm{año}^{-1}\right)$, área basal $\left(\mathrm{ICA}_{\mathrm{g}}, \mathrm{m}^{2} \mathrm{ha}^{-1} \mathrm{año}^{-1}\right)$ $\mathrm{y}$ volumen $\left(\mathrm{ICA}_{\mathrm{v}}, \mathrm{m}^{3} \mathrm{ha}^{-1} \mathrm{año}^{-1}\right)$. Las variables, $d m c, G \mathrm{y}$ $V$ fueron medidas sobre la misma unidad experimental a través de tiempo. Es razonable que los sujetos (parcelas) sean asumidos independientes, aunque las medidas tomadas sobre un mismo sujeto pueden estar correlacionadas, por lo tanto, se utilizó un modelo lineal generalizado para medidas repetidas.

Sea $y_{\mathrm{ijk}}$ la variable observada repetida sobre el i-ésimo tratamiento, de la j-ésima edad y el k-ésimo sujeto (parcela). El modelo se puede escribir como:

$$
y_{i j k}=\mu+\alpha_{i}+\tau_{j+}(\alpha \tau)_{i j}+\gamma_{k}+\beta_{l}+\varepsilon_{i j k}
$$

Donde:

$\mu, \alpha_{i}, \tau_{k},(\alpha \tau)_{\mathrm{ik}}, \gamma_{\mathrm{k}} \mathrm{y} \beta_{l}$ corresponden, respectivamente, a: la media general, el efecto de la poda del nivel $i$, el efecto del raleo del nivel $j$, la interacción (poda $\times$ raleo) del nivel $i j$ al efecto de la edad del nivel $\mathrm{k} y$ al efecto del bloque al nivel 1 .

$\varepsilon_{i j k}$ representa el error aleatorio con distribución normal multivariada $(0, \Sigma)$, donde $\Sigma$ representa al matriz de varianzas y covarianza que contempla heterogeneidad de varianzas entre tratamientos y dependencia a través da las medidas repetidas de los errores.

Los cálculos fueron realizados en un entorno R (2015), utilizando el paquete stats y el gls para testear las diferen- 
cias entre tratamientos y entre edades para ciclo estudiado. Se estimó la matriz de correlación entre las para analizar el grado de asociación entre las variables: precipitación, evapotranspiración potencial y déficit hídrico sobre las variables en estudio ( $d m c, G$ y V).

\section{RESULTADOS}

Efecto del raleo y la poda sobre el crecimiento en diámetro medio cuadrático (dmc). Se encontraron diferencias significativas en $d m c$ a partir de los 8 años para todos los tratamientos en relación al testigo. A partir del año 10 se observan diferencias entre las distintas intensidades de raleo (T2 y T3) y en relación al testigo (T1); efecto que se mantiene a lo largo del ciclo estudiado (cuadro 2 y figura $2 \mathrm{~A}$ ).

El tratamiento testigo (T1) mostró valores de $d m c$ claramente inferiores a los tratamientos con raleo. El T3 que alcanzó un $d m c$ de $30,4 \pm 0,9 \mathrm{~cm}$, resultó un 39,2\% superior al testigo $(21,9 \mathrm{~cm})$. El T2, con un crecimiento levemente inferior $(25,9 \mathrm{~cm})$, fue un $23,9 \%$ mayor que el $\mathrm{T} 1$. A los 12 años, en los tres tratamientos parecería alcanzar su máximo de incremento (figura 3 ).

Cuando se analiza el crecimiento del diámetro a una misma intensidad de raleo (50\%), los tratamientos con y sin poda mostraron tendencias similares $(P=0,9739$ a los
13 años, figura 2B). La merma en el crecimiento a causa de la poda no es significativa y se mantuvo a lo largo del ciclo estudiado. Al año 13 el tratamiento T4 alcanzó un valor de $24,3 \mathrm{~cm}$, que al compararlo con el T2 significó una reducción de sólo el 6,3\% por el efecto de la poda. En el caso del T5 alcanzó un tamaño de $29 \mathrm{~cm}$ lo que equivale a una disminución en relación al T3 de apenas el 4,6\%.

En todos los esquemas propuestos el incremento corriente anual del $d m c\left(\mathrm{ICA}_{d m c}\right.$ ) decrece con la edad y siempre fue menor en el testigo (figura 3). Para el período que comprende desde los 6 a los 13 años de la plantación, el T3 presentó los mayores incrementos. La mayor caída en el ICA $\mathrm{A}_{d m c}$ para todos los tratamientos se dio entre los años 6 y 8 .

Crecimiento en área basal. El tratamiento de raleo afectó significativamente $(P<0,01)$ el área basal desde el momento de su aplicación (figura 4A), resultando el testigo superior a las parcelas raleadas. El área basal al año 6 en el T1 fue de 11,8 $\mathrm{m}^{2} \mathrm{ha}^{-1}$, de 7,3 $\mathrm{m}^{2} \mathrm{ha}^{-1}$ para el $50 \%$ de raleo sin poda (T2) y de $3,4 \mathrm{~m}^{2} \mathrm{ha}^{-1}$ para el $75 \%$ de raleo sin poda (T3). Para este último tratamiento, el valor inicial se redujo a la cuarta parte en relación al testigo.

Si se toma como referencia el valor inicial del área basal de cada tratamiento, el incremento detectado en el tratamiento testigo fue de $230 \%$, en tanto que para el T2, fue

Cuadro 2. Análisis de comparación de medias, para tratamientos de poda y raleo, en un ciclo de 6 a 13 años.

Comparison of means analysis for pruning and thinning treatments, in a cycle of 6 to 13 years.

\begin{tabular}{|c|c|c|c|c|c|c|c|c|c|}
\hline \multirow{2}{*}{ Variable } & \multirow{2}{*}{ Tratamientos } & \multicolumn{8}{|c|}{ Edad (años) } \\
\hline & & 6 & 7 & 8 & 9 & 10 & 11 & 12 & 13 \\
\hline \multirow{5}{*}{$d m c(\mathrm{~cm})$} & $\mathrm{T} 1$ & $\mathrm{a}-\mathrm{A}$ & $\mathrm{a}-\mathrm{B}$ & $\mathrm{a}-\mathrm{B}$ & $\mathrm{a}-\mathrm{BC}$ & $\mathrm{a}-\mathrm{C}$ & $\mathrm{a}-\mathrm{C}$ & $\mathrm{a}-\mathrm{C}$ & $\mathrm{a}-\mathrm{C}$ \\
\hline & $\mathrm{T} 2$ & $\mathrm{a}-\mathrm{A}$ & $\mathrm{a}-\mathrm{B}$ & $b-B$ & $\mathrm{~b}-\mathrm{BC}$ & $b-C$ & $b-C$ & $\mathrm{~b}-\mathrm{CD}$ & $b-D$ \\
\hline & $\mathrm{T} 3$ & $\mathrm{a}-\mathrm{A}$ & $\mathrm{a}-\mathrm{B}$ & b-B & b-B & $\mathrm{c}-\mathrm{C}$ & $\mathrm{c}-\mathrm{CD}$ & $\mathrm{c}-\mathrm{DE}$ & $c-E$ \\
\hline & $\mathrm{T} 4$ & $\mathrm{a}-\mathrm{A}$ & $\mathrm{a}-\mathrm{B}$ & $b-B$ & $\mathrm{a}-\mathrm{B}$ & $\mathrm{ab}-\mathrm{C}$ & $\mathrm{ab}-\mathrm{C}$ & $\mathrm{b}-\mathrm{CD}$ & $b-D$ \\
\hline & T5 & $\mathrm{a}-\mathrm{A}$ & $\mathrm{a}-\mathrm{B}$ & $\mathrm{b}-\mathrm{BC}$ & b-CD & bc-DE & bc-EF & bc-FG & $c-G$ \\
\hline \multirow{5}{*}{$G\left(\mathrm{~m}^{2} \mathrm{ha}^{-1}\right)$} & $\mathrm{T} 1$ & $\mathrm{a}-\mathrm{A}$ & $a-B$ & $\mathrm{a}-\mathrm{B}$ & $\mathrm{a}-\mathrm{B}$ & $\mathrm{a}-\mathrm{BC}$ & $\mathrm{c}-\mathrm{CD}$ & $\mathrm{a}-\mathrm{D}$ & $a-D$ \\
\hline & $\mathrm{T} 2$ & $a b-A$ & $b-B$ & $b-B$ & $\mathrm{~b}-\mathrm{C}$ & $b-C$ & $b-C D$ & $b-D E$ & $b-E$ \\
\hline & T3 & $\mathrm{b}-\mathrm{A}$ & $\mathrm{c}-\mathrm{AB}$ & $c-B$ & $\mathrm{c}-\mathrm{BC}$ & $\mathrm{c}-\mathrm{CD}$ & $\mathrm{c}-\mathrm{DE}$ & $c-E$ & $c-E$ \\
\hline & $\mathrm{T} 4$ & $\mathrm{ab}-\mathrm{A}$ & $\mathrm{b}-\mathrm{AB}$ & $\mathrm{b}-\mathrm{BC}$ & $\mathrm{b}-\mathrm{CD}$ & b-DE & $b-E F$ & $\mathrm{c}-\mathrm{F}$ & $\mathrm{b}-\mathrm{F}$ \\
\hline & T5 & $\mathrm{b}-\mathrm{A}$ & $\mathrm{c}-\mathrm{AB}$ & $\mathrm{c}-\mathrm{B}$ & $c-B$ & $\mathrm{c}-\mathrm{BC}$ & $\mathrm{c}-\mathrm{BC}$ & $c-C$ & $\mathrm{c}-\mathrm{C}$ \\
\hline \multirow{5}{*}{$V\left(m^{3} h a^{-1}\right)$} & $\mathrm{T} 1$ & $\mathrm{a}-\mathrm{A}$ & $\mathrm{a}-\mathrm{B}$ & $\mathrm{a}-\mathrm{B}$ & $\mathrm{a}-\mathrm{BC}$ & $\mathrm{a}-\mathrm{CD}$ & $\mathrm{a}-\mathrm{D}$ & a-DE & $a-E$ \\
\hline & $\mathrm{T} 2$ & $a b-A$ & $\mathrm{ab}-\mathrm{AB}$ & $\mathrm{ab}-\mathrm{BC}$ & $\mathrm{b}-\mathrm{CD}$ & b-DE & b-EF & $\mathrm{b}-\mathrm{FG}$ & $b-G$ \\
\hline & $\mathrm{T} 3$ & $a b-A$ & b-A & $c-A$ & $\mathrm{c}-\mathrm{A}$ & cd-AB & $c-B$ & cd-B & cd-B \\
\hline & $\mathrm{T} 4$ & $\mathrm{a}-\mathrm{A}$ & b-A & bc-AB & $b-B$ & $\mathrm{bc}-\mathrm{BC}$ & bc-CD & bc-DE & $c-E$ \\
\hline & T5 & b-A & b-A & $\mathrm{c}-\mathrm{AB}$ & $c-B$ & $\mathrm{~d}-\mathrm{BC}$ & $\mathrm{c}-\mathrm{CD}$ & d-D & d-D \\
\hline
\end{tabular}

Para una misma edad, letras minúsculas diferentes indican diferencias significativas $(P<0,05)$ entre tratamientos. Para cada tratamiento, letras mayúsculas diferentes indican diferencias significativas entre edades. 

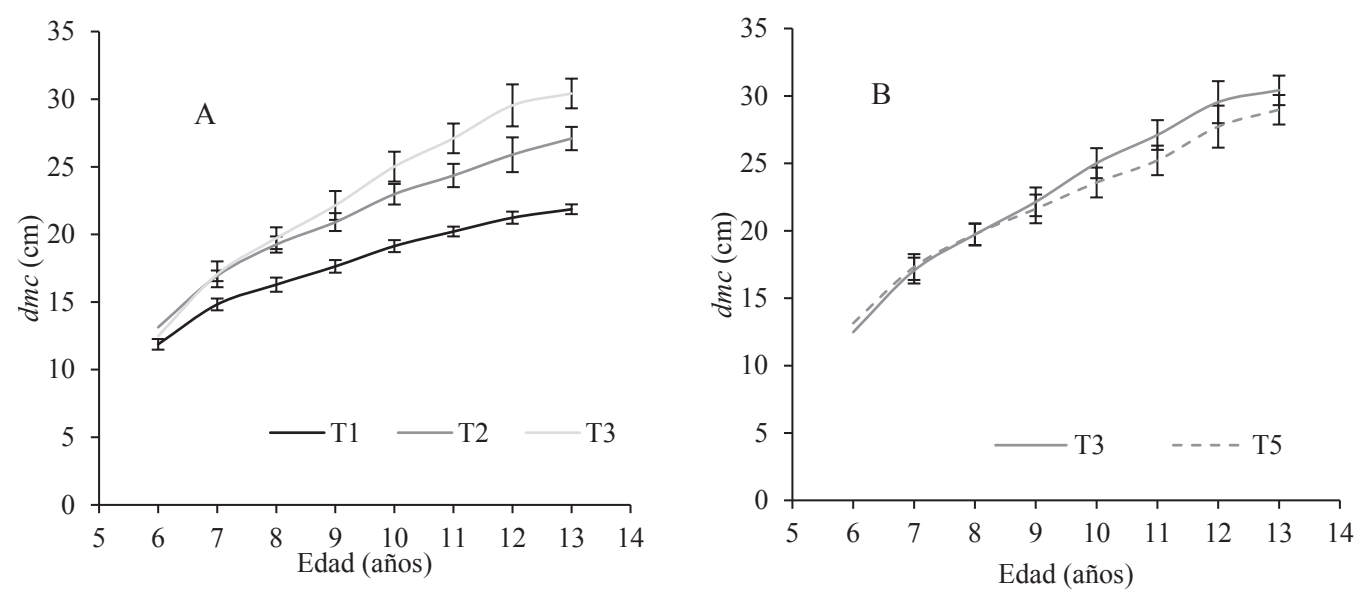

Figura 2. Evolución del diámetro cuadrático medio $(d m c)$ de Pinus radiata $\mathrm{A})$ con distintas intensidades de raleo $(\mathrm{T} 1=\mathrm{Sin}$ raleo, $\mathrm{T} 2$ $=50 \%$ raleo, $\mathrm{T} 3=75 \%$ raleo). B) Tratamientos con $50 \%$ de raleo con (T4) y sin poda (T2). (Las líneas verticales representan los desvíos estándar).

Quadratic mean diameter $(\mathrm{dmc})$ of Pinus radiata. A) With different thinning intensities (T1, without thinning; T2, 50 \% thinning; T3, 75 $\%$ thinning); B) treatments with $50 \%$ thinning, with pruning (T4) and without pruning (T2). (Vertical lines account for standard deviations).

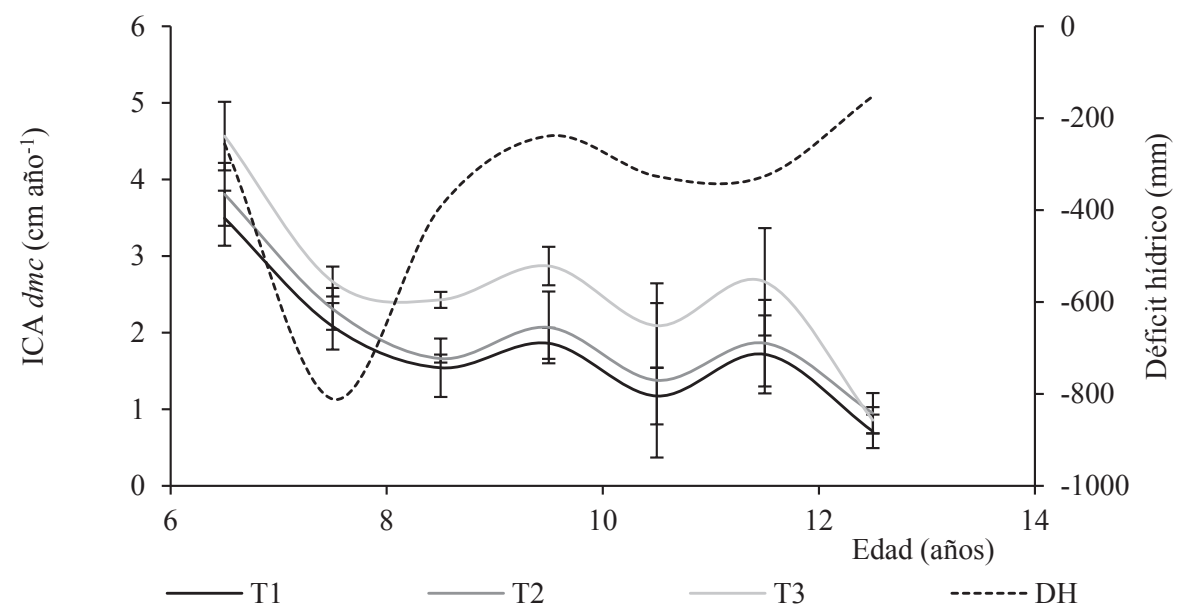

Figura 3. Incremento corriente anual (ICA) en $d m c$ de Pinus radiata para un rodal sometido a distintos tratamientos de poda y raleo. (Las líneas verticales representan los desvíos estándar). En el eje secundario se observa el déficit hídrico DH (mm), obtenido de la diferencia de la ETP, evapotranspiración potencial $(\mathrm{mm})$ y la precipitación $(\mathrm{mm})$. El período de crecimiento comprende el año de septiembre a abril.

Annual current increase in qmd of Pinus radiata for a stand subjected to different thinning and pruning treatments (vertical lines account for standard deviations). The secondary axis shows the water deficit (DH = ETP-PP) for the growth period, which ranges from September to April.

del $311 \%$ y para el T3 fue del $483 \%$. La diferencia en las tasas del crecimiento se debe, partiendo de situaciones iniciales diferentes, a que el incremento en área basal inicial en T1 disminuye ostensiblemente en el primer año, en tanto que para los tratamientos con raleo como por ejemplo $\mathrm{T} 3$, los incrementos se mantienen en niveles similares a lo largo de todo el período estudiado. Para la situación con poda (figura 4B), el crecimiento fue levemente menor $(P=$ 0,97 ) entre el T2 y el T4, de manera análoga a lo ocurrido para el $d m c$.
Los tratamientos T1 y T2 mostraron los mayores ICA, seguido de $\mathrm{T} 3$ y T4, y finalmente el de menor $\mathrm{ICA}_{\mathrm{g}}$ en el tratamiento T5, coincidentemente con la mayor intervención realizada. Las mayores caídas en el ICA $\mathrm{g}$ se presentaron entre los años 6 y 8 (figura 5), y las diferencias entre tratamientos se unifican entre los años 10 y 11 . A los 12 años las mayores diferencias en ICA se plantean entre el tratamiento con $50 \%$ raleo sin poda (T2), respecto de aquellos con $75 \%$ de raleo con (T5) y sin poda (T3). Similar a la tendencia presentada por el $d m c$, durante el 

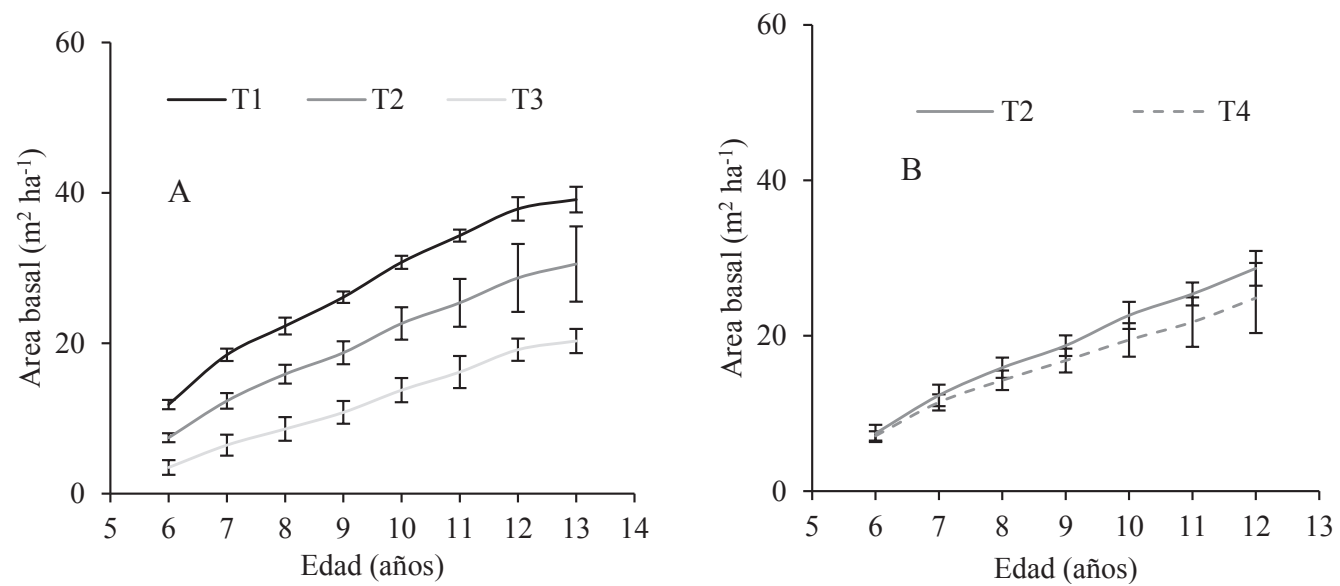

Figura 4. Evolución del crecimiento en área basal por ha de Pinus radiata: $\mathrm{A})$ con distintos tratamientos de intensidades de raleo (T1 = sin raleo, $\mathrm{T} 2=50 \%$ raleo, T3 $=75 \%$ de raleo. B) para el $50 \%$ de raleo, con y sin poda. (Las líneas verticales representan los desvíos estándar).

Evolution of growth in basal area per hectare of Pinus radiata: A) with different thinning intensities (T1, without thinning; T2, $50 \%$ thinning; T3, $75 \%$ thinning); B) treatments with $50 \%$ thinning, with pruning and without pruning. (Vertical lines account for standard deviations).

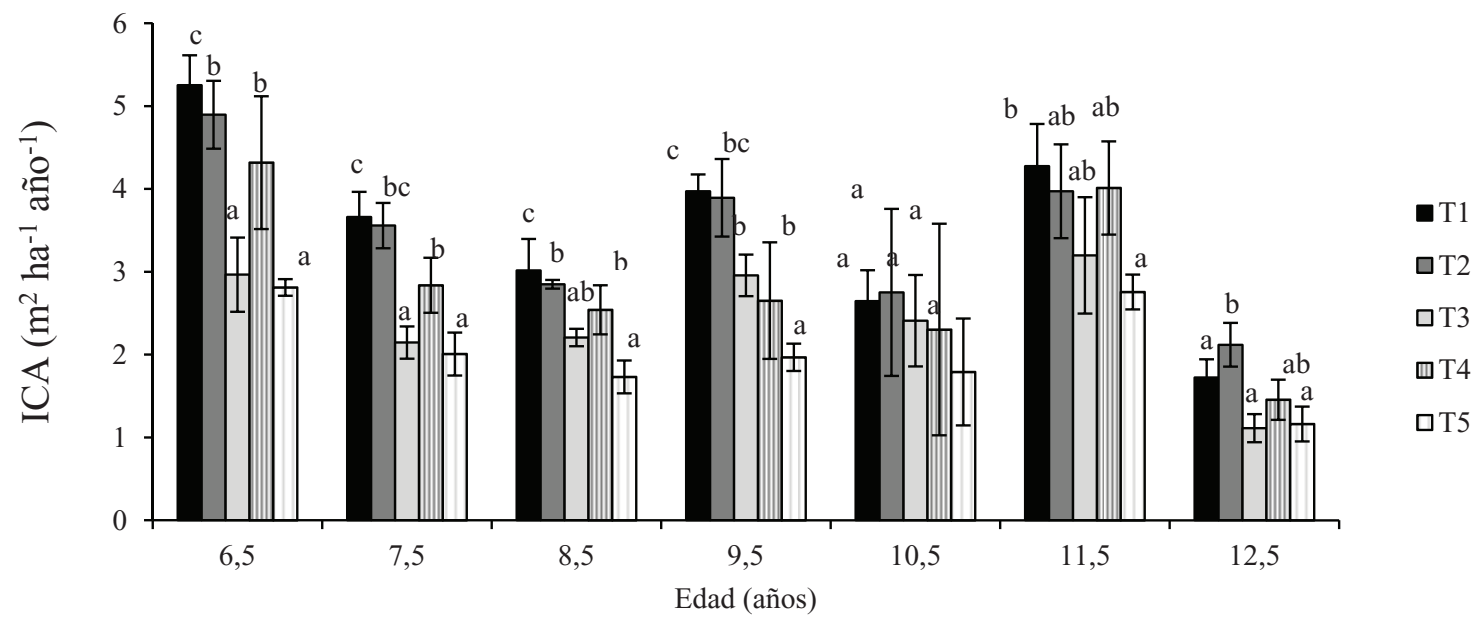

Figura 5. Evolución del incremento corriente anual (ICA) en área basal para los tratamientos en estudio. (Las líneas verticales representan los desvíos estándar).

Evolution of increase in basal area for treatments under study (vertical lines account for standard deviations). Values followed by the same letter per treatment present no significant differences $(P<0.05)$.

período analizado, el ICA mostró una correlación negativa significativa con la evapotranspiración del período de crecimiento $(\mathrm{r}=-0,67, P<0,01)$.

Crecimiento en volumen. A los 13 años, el tratamiento testigo contó con un volumen de $189,7 \mathrm{~m}^{3} \mathrm{ha}^{-1}$ lo cual significa una tasa de $14,6 \mathrm{~m}^{3}$ ha $^{-1} \mathrm{año}^{-1}$. Los tratamientos con una intensidad de raleo del $50 \%$ (T2 y T4), sólo mostraron una caída significativa en volumen a los 13 años, donde el T2 alcanzó los 161,3 $\mathrm{m}^{3} \mathrm{ha}^{-1}$, mientras que en el T4, fue de $139,5 \mathrm{~m}^{3} \mathrm{ha}^{-1}$; es decir se redujo significativamente $(13 \%)$ en relación a la misma densidad sin poda (cuadro 2 , figura 6).
Para la intensidad de raleo del $75 \%$ (T3 y T5), no se encontraron diferencias significativas en el volumen del rodal a lo largo del ciclo estudiado. Las diferencias entre los tratamientos T2 y T3 (50 y $75 \%$ de raleo, sin poda) comienzan a observarse al año 8 y se mantienen hasta el año 13 , alcanzando un valor de $161,3 \mathrm{~m}^{3} \mathrm{ha}^{-1} \mathrm{y}$ de $108 \mathrm{~m}^{3}$ ha $^{-1}$, respectivamente (figura 6).

Para los tratamientos con poda la tendencia general fue una mayor acumulación de volumen en el tratamiento con $50 \%$ de raleo (T4) en relación al T5 (75\% de raleo), a excepción del año 11.

Los incrementos en volumen (figura 7) tuvieron un comportamiento similar al incremento en área basal con un 

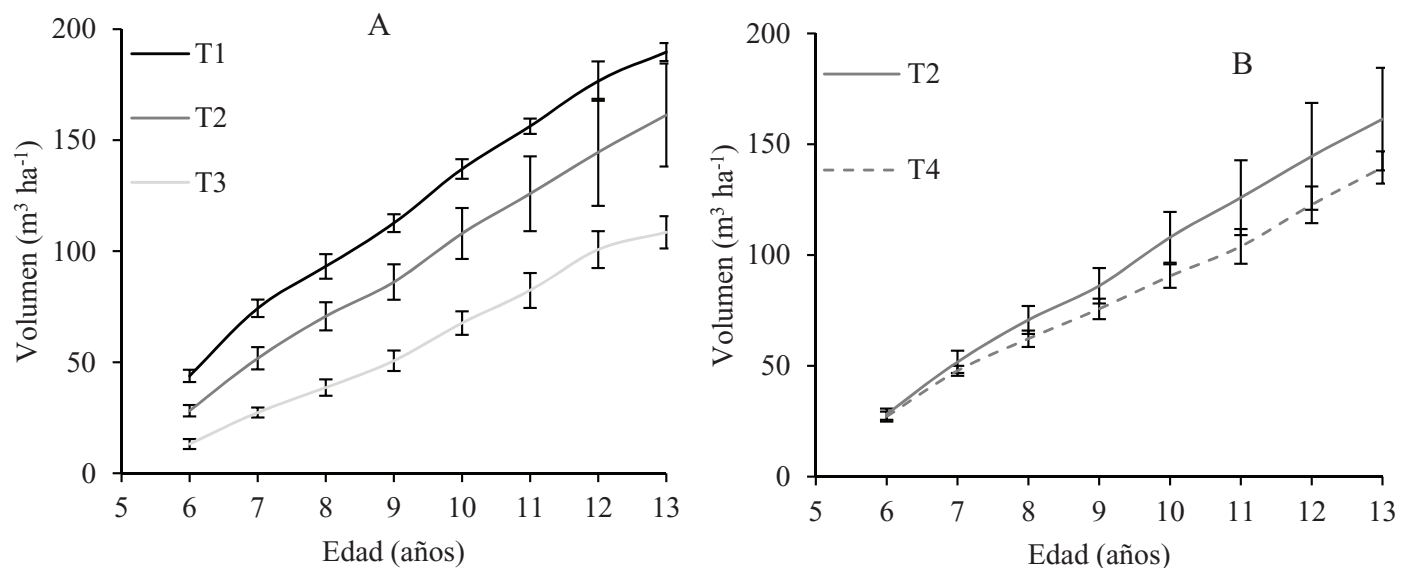

Figura 6. Evolución del crecimiento en volumen por hectárea de Pinus radiata A) para distintos tratamientos de raleo (50 y $75 \%)$ y B) para el tratamiento testigo y $50 \%$ de raleo, con y sin poda.

Evolution of growth of volume per hectare of Pinus radiata. A) For different thinning treatments, and B) for control treatment and treatment with $50 \%$ thinning, with and without pruning.

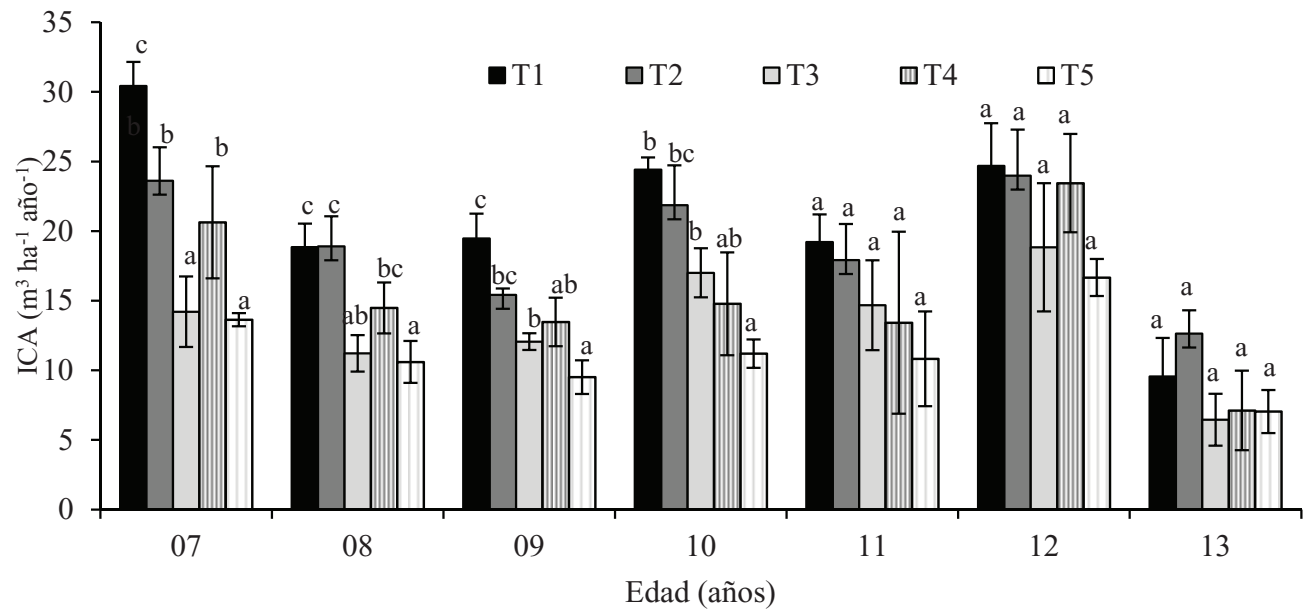

Figura 7. Incremento corriente anual (ICA) en volumen de acuerdo a los tratamientos aplicados (las líneas verticales representan los desvíos estándar). Letras distintas denotan diferencias significativas $(P<0,05)$.

Annual current increase in volume, based on treatments applied (vertical line represents standard deviations). Values followed by the same letter per treatment present no significant differences $(P<0.05)$.

manifiesto descenso del crecimiento entre los años 7 y 9. A partir de los 10 y 11 años, las diferencias estadísticas dejan de ser significativas entre tratamientos de poda y raleo.

Crecimiento de los árboles dominantes. En el cuadro 3 se muestran los estimadores de los indicadores dasométricos de los árboles dominantes a la edad de 13 años (200 árboles ha $\left.^{-1}\right)$. En comparación con los demás tratamientos, el T3 (75\% de raleo, sin poda) mostró los mejores crecimientos, tanto a nivel de árbol individual como de rodal. En efecto, el $d m c$ de los árboles dominantes alcanzó un valor de $32 \mathrm{~cm}$ y representa el $79 \%$ del área basal y el $80 \%$ del volumen en relación a los valores de las varia- bles que consideran el total de los individuos por hectárea (cuadro 3). Por otro lado, para la misma cantidad de árboles se observa que en el T3, con $75 \%$ de raleo se logra una mayor concentración de volumen en comparación al T1. En cuanto al T2 y T5, no fueron significativamente distintos al T3 en las variables analizadas, pero aumentaron significativamente en relación a T1. Se observa que T2 solo acumula el $46 \%$ del volumen en los árboles dominantes en comparación al total por ha, valor muy inferior al señalado para T3 y T5. En cuanto al T4 (50 \% de raleo con poda) el crecimiento de las variables analizadas no mostró diferencias con el testigo, es decir los árboles dominantes presentaron similar $d m c$ y área basal. 
Cuadro 3. Variables dasométricas para Pinus radiata al año 13 de los 200 árboles dominantes en tratamientos de poda y raleo. Letras distintas denotan diferencias significativas $(P<0,05)$.

Dasometric variables of Pinus radiata stand corresponding to 200 dominant trees in pruning and thinning treatments. Standard deviations shown between brackets. Values followed by the same letter per treatment present no significant differences $(P<0.05)$.

\begin{tabular}{cccc}
\hline Tratamiento & $d m c(\mathrm{~cm})^{*}$ & Área basal $\left(\mathrm{m}^{2} \mathrm{ha}^{-1}\right)$ & Volumen $\left(\mathrm{m}^{3} \mathrm{ha}^{-1}\right)$ \\
\hline T1 & $26,5( \pm 1,0) \mathrm{a}$ & $11,1( \pm 0,9) \mathrm{a}$ & $55,7( \pm 5,24) \mathrm{a}$ \\
$\mathrm{T} 2$ & $30,0( \pm 1,7) \mathrm{bc}$ & $14,2( \pm 1,6) \mathrm{bc}$ & $74,7( \pm 10,3) \mathrm{bc}$ \\
$\mathrm{T} 3$ & $32,0( \pm 1,2) \mathrm{c}$ & $16,1( \pm 1,3) \mathrm{c}$ & $87,1( \pm 8,2) \mathrm{c}$ \\
$\mathrm{T} 4$ & $28,2( \pm 1,2) \mathrm{ab}$ & $12,5( \pm 1,1) \mathrm{ab}$ & $64,6( \pm 7,0) \mathrm{ab}$ \\
$\mathrm{T} 5$ & $30,1( \pm 0,7) \mathrm{bc}$ & $14,3( \pm 0,7) \mathrm{bc}$ & $75,4( \pm 4,4) \mathrm{bc}$ \\
\hline
\end{tabular}

*Valor promedio \pm desvío estándar.

\section{DISCUSIÓN}

La productividad máxima del rodal alcanzó los $14,6 \mathrm{~m}^{3}$ $\mathrm{ha}^{-1}$ año $^{-1}$, similar a lo reportado por Bouillard (1972) en San Clemente de Tuyú $\left(13,3 \mathrm{~m}^{3} \mathrm{ha}^{-1}\right.$ año $\left.^{-1}\right)$. Estos autores también mencionan para la zona de Mar de Ajó los incrementos volumétricos variaron entre 9,5 y $30 \mathrm{~m}^{3} \mathrm{ha}^{-1}$ año $\mathrm{O}^{-1}$, indicando que el sitio en estudio serían los de más baja calidad. El crecimiento del tratamiento testigo resultó similar al reportado por Romanyà y Vallejo (2004) en España, e inferior a la media de los sitios más productivos estudiados por Álvarez et al. (2013) en Chile para similares edades. Estos autores reportan una productividad que pueden llegar a $34 \mathrm{~m}^{3} \mathrm{ha}^{-1}$ año ${ }^{-1}$ de incremento medio anual.

La adopción de un esquema de manejo con podas y raleo tempranos implica una pérdida deliberada en volumen, que se debería compensar con la calidad superior y mayor valor de la madera producida, objetivo de producción fuertemente ligado a condiciones económicas y de mercado (Gerding 1991, Espinosa et al. 1994). Como respuesta a la intensidad del raleo aplicado en el presente trabajo, el diámetro de los árboles se incrementó en todos los tratamientos, particularmente en los mayores espaciamientos ( $75 \%$ raleo). Espinosa et al. (1994) también reportan el efecto positivo del raleo en el $d m c$, al comparar las situaciones con y sin raleo. Las diferencias entre tratamientos con y sin raleo también se detectaron en área basal y volumen. Cuando el raleo fue del $75 \%$, el volumen total se redujo a la mitad, tal como lo indican Espinosa et al. (1994). Es de destacar que la densidad inicial del trabajo de referencia fue de 1.550 plantas ha- ${ }^{-1}$, un $39 \%$ superior al del presente trabajo, mientras que el incremento medio anual fue de un $23 \%$ superior en aquel trabajo, en similares períodos de crecimiento.

El efecto positivo del raleo sobre el crecimiento en diámetro se atribuye a una reducción en la competencia por luz, agua y nutrientes (Smith 1997). Con una intervención del $50 \%$, al remover los individuos competidores se logró un incremento de 5,2 cm en el diámetro (T1 vs T2); valor similar al mencionado por Schoelzke (2003). El incremento en diámetro fue mayor $(8,6 \mathrm{~cm})$ con una intensidad de raleo al $75 \%$. Navarro et al. (2010) trabajando con Pinus halepensis con raleos aplicados luego de 8-9 años de la plantación, sostienen que el efecto sobre el crecimiento en diámetro, disminuye con el tiempo manteniendo la tendencia aún con la presencia de plagas o lluvias insuficientes.

Cuando se analiza el efecto de la poda, se observa que tanto para el $50 \%$ como para el $75 \%$ de raleo no hubo una reducción significativa del diámetro a nivel de árbol individual ni en el área basal (cuadro 2, figuras 2 y 4). Esto concuerda con Sutton y Crowe (1975), quienes plantean para la misma especie que intensidades de poda menores al $50 \%$ no afectan el crecimiento. Sin embargo, si se analiza en términos relativos significó una reducción en la productividad alcanzó del 13-14 \% en volumen (para T2 vs T4 y T3 vs T5). Algunos autores plantean que intensidades de poda entre $25-40 \%$ de la copa en coníferas puede afectar negativamente el crecimiento en diámetro. La eliminación de la parte inferior de la copa en árboles relativamente jóvenes creciendo en situación de baja competencia podría significar la remoción de tejido aun activo en la parte inferior del fuste que contribuye al crecimiento en forma sustancial (O'Hara 1991, Makinnen et al. 2014), lo que llevaría a obtener árboles más cilíndricos (Pinkard y Beadle 2000), aspecto no contemplado en nuestro trabajo. Para intensidades mayores al $50 \%$ de poda que pueden afectar significativamente el incremento en diámetro de los árboles, Nielsen y Pinkard (2003) postulan que esto puede significar una ventaja por la generación de ramas de menores diámetros con oclusiones de poda más pequeñas y tempranas, aspectos no evaluados en el presente trabajo. Las observaciones realizadas indican que hasta el año 13, aún no se produjo la oclusión de la herida de la última poda realizada cuatro años atrás. En base a nuestras observaciones sería interesante evaluar la realización de podas a edades más tempranas (antes de los 6 años), con intensidades del $40 \%$. Podría resultar una alternativa para evitar la formación de grandes ramas que demoran más de tres años en ocluir. Es de esperar que la mejora en la calidad de la madera por ausencia de defectos justifique su aplicación en los primeros años de establecimiento del rodal. 
El análisis de los árboles dominantes permite obtener una información complementaria de los individuos del rodal con mejor potencial de crecimiento. En nuestro estudio se observa que los tratamientos más intensamente raleados tuvieron un marcado efecto en la acumulación de volumen, contrariamente a lo observado por Espinosa et al. (1994), quienes no reportan modificaciones en los árboles dominantes. La menor producción volumétrica en el tratamiento de $75 \%$ de raleo en comparación con $50 \%$ resulta significativa aún a los 13 años. Por lo tanto, ante la ausencia de diferencias significativas entre $\mathrm{T} 2$ y T3, tanto en la evaluación a nivel de árbol individual $(d m c)$ como de rodal (área basal y volumen), sería recomendable la aplicación de intensidades de raleos no superiores del $50 \%$.

La similitud entre T3 y T5 indicaría que no hay efecto de la poda sobre el crecimiento de los dominantes, por lo tanto, la remoción del $40 \%$ de la copa estaría mejorando la calidad de la madera y aumentando la concentración del crecimiento en los individuos remanentes. Sin embargo, esta mayor concentración de madera en los árboles dominantes, conlleva una pérdida significativa de volumen maderable en relación al $50 \%$ de raleo, cuya incidencia económica no se ha analizado en el presente estudio.

Los resultados sugieren que en el caso de los árboles dominantes no habría diferencias en la producción en volumen entre intensidades de raleo o bien de estos con el tratamiento que combina la poda con $75 \%$ de intensidad de raleo (cuadro 3). En consecuencia, la decisión de la aplicación de la poda se basaría en una cuestión de mercado; esto es, si reconoce la calidad de la madera. Por otro lado, la reducción de niveles de competencia disminuye el riesgo de ataque de plagas tales como Sirex noctilio Fabricius (Hymenoptera-Siricidae).

La aplicación de raleos tempranos genera una subocupación inicial del sitio que podría complementarse con la actividad ganadera (Peri et al. 2002, Sotomayor 2009) y posiblemente generaría una mayor aceptación por parte de los productores de la región.

En cuanto al efecto negativo del déficit hídrico sobre el crecimiento, este se explica por la relación significativa encontrada entre la evapotranspiración acumulada en el período de crecimiento que va de septiembre a marzo (durante todos los años del ciclo analizado) y la caída del $d m c$. Entre los años 7-8, donde se detectó una caída del $\mathrm{ICA}_{d m c}$, se produjo el mayor déficit hídrico, con precipitaciones que alcanzaron los $551 \mathrm{~mm}$, representando una disminución del $38 \%$ de la media histórica de la región $(885 \mathrm{~mm})$.

El impacto sobre el crecimiento es esperable dado que las plantaciones se localizan en suelos con escasa profundidad efectiva donde la capacidad de almacenaje se reduce sustancialmente. Se presume que los tratamientos más densos, con mayor demanda de agua por unidad de superficie, debieron acentuar la competencia en relación a los tratamientos intervenidos (Huber y Trecaman 2004). Similares resultados son reportados por Gerding y Schlatter (1995) cuando analizaron el efecto de las precipitaciones sobre el incremento en volumen. La similitud en los incrementos corrientes anuales en área basal y volumen entre los tratamientos al final del ciclo (figuras 6 y 7) pueden deberse a un efecto combinado de la edad con condiciones climáticas favorables. Los años 9 y 11 fueron los de menor déficit hídrico y las precipitaciones fueron similares o superiores al promedio.

\section{CONCLUSIONES}

La aplicación de un raleo en una plantación de Pinus radiata en el sudeste de la provincia de Buenos Aires, afectó el crecimiento de los árboles individuales y de la masa. La intensidad de raleo del $50 \%$ a los seis años de establecimiento de una plantación es el más recomendado dado que permite combinar crecimiento a nivel de árbol individual con una acumulación importante de volumen en los árboles remanentes.

Si bien la remoción de la copa en un $40 \%$ con $50 \%$ de raleo implica una pérdida productiva, se debería analizar si esta pérdida se compensa con la obtención de madera con menos defectos. Adicionalmente, la poda posibilita una mayor entrada de luz al estrato inferior de la plantación lo que genera un ambiente propicio para el desarrollo de especies forrajeras con vistas al establecimiento de un sistema silvopastoril. Los crecimientos promisorios obtenidos en estos suelos de bajo índice de productividad indican que es posible destinar estos sitios a la producción de madera de calidad, que cobra importancia en un ámbito de creciente agriculturización de la región. Considerando que son suelos con un uso actual marginal, el desarrollo de plantaciones con vistas a la producción de madera para aserrado requiere de la aplicación de un manejo forestal intensivo, que contemple no solo los tratamientos intermedios de poda y raleo descriptos en el presente estudio, sino también, el uso de plantines de calidad con una genética de avanzada, adecuadas técnicas de establecimiento y un aprovechamiento acorde con el objetivo inicial propuesto.

Del presente estudio se desprende la necesidad de avanzar en otros esquemas de poda que contemplen la oportunidad y la intensidad de las intervenciones y un análisis económico financiero que determine la conveniencia o no de este manejo para la región.

\section{AGRADECIMIENTOS}

El trabajo de campo ha sido parcialmente financiado por el Proyecto de INTA PNFOR 1104073 "Bases silvícolas para sustentar la productividad de las plantaciones y los recursos del ambiente" y por el PIA 10052 (BIRF Componente 2 Plantaciones Forestales Sustentables).

\section{REFERENCIAS}

Álvarez J, HL Allen, TJ Albaugh, JL Stape, BP Bullock, C Song. 2013. Factors influencing the growth of radiata pine planta- 
tions in Chile. Forestry 86(1): 13-26.

Barrio M, F Castedo, J Majada, A Hevia. 2009. Manual básico de la poda y formación de los árboles forestales. Madrid, España. Editorial Mundi-Prensa. 255 p.

Bravo F, JG Álvarez González, MD Rio, M Barrio, JA Bonet Lledos, A Bravo Oviedo, J Vázquez Piqué. 2011. Growth and yield models in Spain: historical overview, contemporary examples and perspectives. Forest Systems 20(2): 315-328.

Bouillard, M.A. 1972. El crecimiento de Pinus radiata, Pinus pinaster y $P$. halepensis en las dunas de San Clemente del Tuyú y Mar de Ajó, Provincia de Buenos Aires. Revista Forestal Argentina 16(3): 97-101.

Costas R, P Mac Donagh, E Weber, P Irschick, J Palavecino. 2002. Efectos de la densidad de plantación y la altura de poda sobre la producción de Pinus taeda L. a los 5 años de edad. Revista Floresta 33(1): 79-87.

Costas R, P Mac Donagh, E Weber, S Figueredo, P Irschick. 2005. Influencias de la densidad y podas sobre la producción de Pinus taeda L. a los 7 años de edad. Ciência Florestal 15(3): 275-284.

Costas R, S Korth, S Figueredo, E Weber, P Mac Donagh, A Friedl, J Heck, YM Alves Do Porto. 2007. Sistema de ecuaciones de producción y crecimiento de Pinus taeda L. con variables asociadas a las podas. Investigación Agraria: Sistemas y Recursos Forestales 16(2): 136-144.

Crechi EH, RA Friedl, HE Fassola, RA Fernandez, JC Fahler. 2001. Efectos de la Intensidad y Oportunidad de Raleo en Pinus taeda L. sobre el crecimiento y la producción en el noroeste de Misiones, Argentina. In $2^{\circ}$ Simpósio LatinoAmericano sobre Manejo Florestal. Santa María, RS, Brasil. 13 e 14 de setembro. p. 189-210.

Espinosa BM, J García, V Osvaldo. 1994. Efecto de intensidades diferentes de raleo en el crecimiento de un rodal de Pinus radiata. Bosque 15(1): 55-65.

Fassola HE, FA Moscovich, P Ferrere, F Rodríguez. 2002. Evolución de las principales variables de árboles de Pinus taeda L. sometidos a diferentes tratamientos silviculturales en el nordeste de la provincia de Corrientes, Argentina. Ciência Florestal 12(2), 51-60.

Fassola HE, SM Lacorte, AN Pachas, A.E Keller. 2004. Experiencias sobre manejo silvopastoril en Misiones y NE de Corrientes, Argentina. In $11^{\text {a }}$ Jornadas Técnicas Forestales y Ambientales. 7 al 9 de octubre. Eldorado, Misiones, Argentina. Facultad de Ciencias Forestales UNaM. Eldorado, Misiones, Argentina. 14 p.

García O. 1993. Stand growth models: Theory and practice. In Advancement in Forest Inventory and Forest Management Sciences. Proceedings of the IUFRO Seoul Conference. Forestry Research Institute of the Republic of Korea. p. 22-45.

Gerding V. 1991. Manejo de las plantaciones de Pinus radiata D. Don en Chile. Bosque 12(2): 3-10.

Gerding V, JE Schlatter. 1995. Variables y factores del sitio de importancia para la productividad de Pinus radiata D. Don en Chile. Bosque 16(2): 39-56.

Golfari L. 1959. Necesidades climáticas del Pinus radiata D. Don. (=P. insignis Dougl). Revista Forestal Argentina 3 (3): 77-84.

Groot A, R Schneider. 2011. Predicting maximum branch diameter from crown dimensions, stand characteristics and tree species. The Forestry Chronicle 87 (4): 542-551.
Huber A, R Trecaman. 2004. Eficiencia del uso del agua en plantaciones de Pinus radiata en Chile. Bosque 25(3): 33-43.

Jackson DS, HH Gifford. 1974. Environmental variables influencing the increment of radiata pine. 1. Periodic volume increment. New Zeland Journal Forestry Science 4 (1): 3-26.

Jackson DS, HH Gifford, J Chittenden. 1975. Environmental variables influencing the increment of Pinus radiate. 2. Effects of seasonal drought on height and diameter increment. New Zeland Journal Forestry Science 5(3): 265-286.

Mäkinen H, E Verkasalo A Tuimala. 2014. Effects of pruning in Norway spruce on tree growth and grading of sawn boards in Finland. Forestry 87 (3): 417-424.

Mead D. 2013. Sustainable management of Pinus radiata plantations. Roma, Italia. FAO. 257 p. (Forestry paper $\mathrm{N}^{\circ} 170$ ).

Meneses M, S Guzmán. 2000. Análisis de la eficiencia de la silvicultura destinada a la obtención de madera libre de nudos en plantaciones de pino radiata en Chile. Bosque 21(2): 8593.

Merino A, C Rey, J Brañas, R Rodriguez-Soalleiro. 2003. Biomasa arbórea y acumulación de nutrientes en plantación de Pinus radiata D. Don en Galicia. Investigación Agraria: Sistemas y Recursos Forestales 12 (2): 85-98.

Navarro FB, MN Jiménez, EM Cañadas, E Gallego, L Terrón, MA. Ripoll. 2010. Effects of different intensities of overstorey thinning on tree growth and understory plant-species productivity in a semi-arid Pinus halepensis Mill. afforestation. Forest Systems 19(3): 410-417.

Neilsen WA, EA Pinkard. 2003. Effects of green pruning on growth of Pinus radiata. Canadian Journal of Forest Research 33: 2067-2073.

O'Hara KL. 1991. A biological justification for pruning in coastal Douglas-fir stands. Western Journal of Applied Forestry 6(3): 59-63.

Peri PL, EG Mason, KM Pollock, AC Varella, DJ Mead. 2002. Early growth and quality of radiata pine in a silvopastoral system in New Zealand. Agroforestry Systems 55(3): 207219.

Pinkard EA, CL Beadle. 2000. A physiological approach to pruning. International Forestry Review 2 (4): 295-305.

Rodríguez R, M Espinosa, G Hofmann, M Marchant. 2003. Needle mass, fine root and stem wood production in response to silvicultural treatment, tree size and competitive status in radiata pine stands. Forest Ecology and Management 186: 287-296.

Romanyà J, VR Vallejo. 2004. Productivity of Pinus radiata plantations in Spain in response to climate and soil. Forest Ecology and Management 195(1): 177-189.

R Development Core Team, 2015. R "A language and environment for statistical computing" (version 3.1.0). Vienna: R Foundation for Statistical Computing. Available in: http:// www.R-project.org/. [9 march 2015].

Schoelzke. 2003. La poda en una plantación de Pinus elliottii. Quebracho 10: 26-38.

Sotomayor A. 2009. Sistemas silvopastoriles, alternativa productiva para un desarrollo sustentable de la agricultura en Chile. In Proceedings Congreso Nacional de Sistemas Silvopastoriles. 14 - 16 de mayo de 2009. Posadas, Misiones, Argentina.

Smith DM, BC Larson, MJ Kelty, PMS Ashton. 1997. The Practice of Silviculture: Applied Forest Ecology (9th Edition). New York, USA. Wiley and Sons. 537 p. 
Sutton WRJ, JB Crowe. 1975. Selective pruning of radiata pine. New Zeland Journal Forestry Science 5: 171-195.

Trincado G, HE Burkhart. 2009. A framework for modeling the dynamics of first-order branches and spatial distribution of knots in loblolly pine trees. Canadian Journal of Forest Re- search 39 (3): 566-579.

Zhang S, HE Burkhart, RL Amateis. 1997. The influence of thinning on tree height and diameter relationships in loblolly pine plantations. Southern Journal of Applied Forestry 21 (4): 199-205.

Recibido: 05.01.15

Aceptado: 20.07.15 